\title{
Determination of Critical Pattern of 60 Meter Ship Construction Project Using Precedence Diagram Method (PDM)
}

\author{
Siswo Hadi Sumantri ${ }^{1}$, Suyono Thamrin ${ }^{2}$, I Nengah Putra Apriyanto ${ }^{3}$, Edi \\ Suhardono $^{4}$, Beny Rudiawan ${ }^{5}$, Arica Dwi Susanto ${ }^{6}$ \\ ${ }^{12345}$ Indonesia Defense University \\ Sentul, Sukahati, Citeureup, Bogor, West Java, Indonesia 16810 \\ ${ }^{6}$ Indonesian Naval Technology College, STTAL. \\ Bumimoro-Morokrembangan, Surabaya 60187, Indonesia
}

\begin{abstract}
PT. XYZ in Surabaya is engaged in the production of commercial ships, providing ship repair and maintenance services as well as general engineering with specifications based on orders. Along with the development of the technology industry, ship production requires every shipyard to evaluate the system used. The time delay is the problem of building a 60 meter commercial ship. The Precedence Diagram Method (PDM) is a method for scheduling project time and showing critical trajectories of project activities. The results showed that in the project scheduling, there are $\mathbf{1 3}$ activities that have a zero value or critical activity which are activities Hull Fabrication, Hull Sub Assembly, Hull Assembly, Hull Erection, Blasting and Painting Raw Material, Finishing Hull, Anode, Machinery Outfitting, Cabling, Computing and Information, Machinery Commisioning, Equipment Commisioning, Harbour Acceptance Test, Yard Trial, Delivery to Customer So that it needs more strict control and supervision from the contractor in carrying out the activities of the 60 meter commercial ship building project activities for each activity/activity so that it does not experience delays and the project can be completed on time.
\end{abstract}

Keywords: Controlling, Planning, Critical trajectories, PDM.

\section{INTRODUCTION}

PT. XYZ in Surabaya is engaged in the production of commercial vessels, providing ship repair and maintenance services as well as general engineering with specifications based on orders (Silvianita, 2018).

Along with the development of the technology industry, ship production requires every shipyard to evaluate the system used. The new ship building project is required to work fast according to the schedule. Poor performance, low quality, and cost overruns often occur in a project (Arica Dwi Susanto, 2018).

The literature used in this paper were obtained to support the research. These literature includes paper titled A fuzzy pert approach to evaluate plant construction project scheduling risk under uncertain resources capacity (R.Lin, 2009). Earned Value Management Systems: Challenges and Future Direction (Wilson, 2013). CPM, PERT and Project Management With Fuzzy Logic Technique and Implementation On A Business (Mazlum, 2015). Integration of Building Information Modeling and Critical Path Method Schedules to Simulate the Impact of Temperature and Humidity at the Project Level (Shan, 2014). Comparative Study of Management Operation System Techniques (MOST) and CPM in Construction Scheduling (Shailla, 2014). Extension of Time Determination in Construction Projects in Nigeria: The Critical Path Method (Andawei, 2014). Critical path analysis for the management of fractured neckof femur (Balla, 1995). The Critical Path Method In Estimating Project Duration (Nafkha, 2016). Critical Path Analysis for New Product Planning (Wong, 1964). Measuring the Actual Energy Cost Performance of Green Buildings: A Test of the Earned Value Management Approach (Dwaikat, 2016). Cost Control and Performance Review of Software Projects by 
Using the Earned Value Management (Alecu, 2014). Critical Path Method in Designing Feasible Solutions (Agarwal, 2013). Traditional Critical Path Method versus Critical Chain Project Management: A Comparative View (M, 2015). Project Planning And Control With Pert And Cpm (K.K.Khandelwal, 2002). Project Planning And Scheduling Using PERT And CPM Techniques With Linear Programming: Case Study (Agyei, 2015). Fast Missile Boat Project Planning using CPM and What If Analysis Method (Silvianita, 2018). CPM Schedule Summarizing Function of the Beeline Diagram Method (Kim, 2012). Earned value method as a tool for project control (Czarnigowska, 2008). The Factors Affecting The Methods of Construction Projects Scheduling: An State of The Art and Overview (Fatemeh Nouban, 2017). Contruction Project Scheduling with Time, Cost and Material Restrictions Using Fuzzy Mathematical Models and Critical Path Method (Daniel Castro-Lacouture, 2009). Planning and Monitoring of industrial punch development processes (Y. Arslan, 2017)

PDM is a network that is generally in the form of a rectangle, while the arrow is only as a guide to the relations of the activities in question. The activity relationship shows the relationship between activities with one line from the previous node to the next node.

This Paper is organized as follows. Section 2 review about the basic ship theory. Section 3 gives result and section 4 discussion of research. Finally, in section 5 present conclusion this paper.

\section{MATERIALS AND METHODS}

Technical Concept factors:

In determining the exact duration

1. Volume of work

2. The condition in project fields

3. Weather conditions

4. Resources conditions (labor, materials, equipment)

5. Experiences on previous project (similar project)

\section{Project Time Management}

There are three stages to be performed in project management:

\section{Planning Process}

Planning process includes the setting of goals, defining projects and forming team organizations. Regarding the capability in working on several projects at once (mostly in large companies), an effective way to assign labors and physical resources is through the project organization. The project organization is led by a project manager who coordinates project activities with other departments and reports to top management.

\section{Scheduling}

Scheduling is the link between labor, money, and materials used in a project. Project scheduling involves specifying the duration of project activities to be completed, raw materials, labor and time required for each activity. The popular approach used is the Gantt Chart or the Bar Chart Method. Other project scheduling methods are PERT (Project Evaluation and Review Technique) and CPM (Critical Path Method).

\section{Controlling}

Project controls include control over resources, cost, quality and budget. Project control is also used to revise the project plan and allow to change/shift resources to a needed places (rearranging) so that the time and cost can be appropriate. Project control involves strict supervision of resources, cost, quality and budget. Control also means the use of feedback loops to revise the project plan and resource arrangements where needed.

\section{Network Component}

There are two approaches to describe the project network: activity on node - AON and activity on arrow (AOA). At the AON convention, the point indicates the activity, whereas at the AOA, the arrow indicates the activity.

\section{Precedence Diagram Method (PDM)}

Precedence Diagram Method is a networking method that is included in the classification of AON (Activity On Node). In this method, the activity is written in a node that is generally rectangular, while the arrows 
as a pointer relationship between the activities concerned. Thus the dummy which is an important sign to indicate the dependence relationship, is not required in PDM.

The precedence method diagram can be described as four relations of activity/logic, Each node has two ends, namely the beginning or beginning $=(\mathrm{S})$ and the end or end $=(\mathrm{F})$ that is (Tan, 1998):

In the precedence diagram method, the existence of four relations of activity/logic can be described. Each node has two ends namely the beginning/starting tip $=(\mathrm{S})$ and end/finish tip $=(\mathrm{F})($ Tan, 1998):

1. Activity relation of Start-to-start (SS): When A starts, B can be started.

2. Activity Relation of Start-to-finish (SF): When A starts, B can be finished.

3. Activity Relation Finish-to-Start (FS): When A finish, B can be started.

4. $\quad$ Activity Relation Finish-to-finish (FF): When A finish, B can be finished.

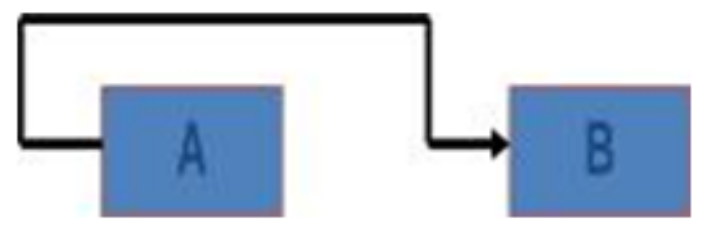

Fig. 1 Activity Relation of Start-to-Start (SS)

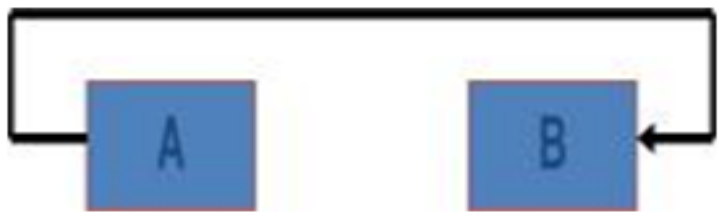

Fig. 2 Activity Relation of Start-to-Finish (SF)

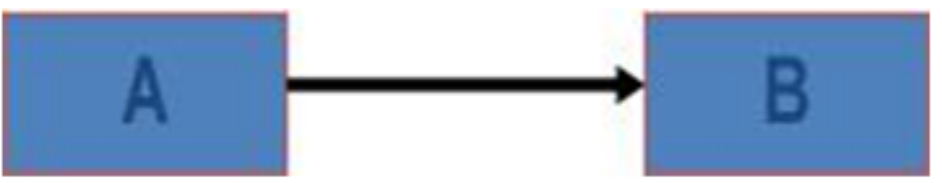

Fig. 3 Activity Relation of Finish-to-Start (FS)

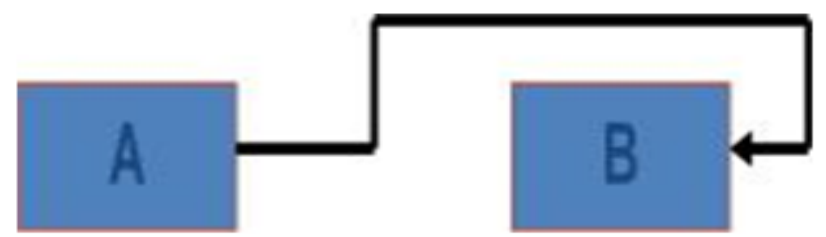

Fig. 4 Activity Relation of Finish-to-Finish (FF)

\section{Method of Research.}

Analysis of this research data are as follows:

Calculations was performed using excel software whose results can handle planning an activity, organizing and controlling time and costs that convert input data into an output data according to purpose, then the overall time of building a 60 meter commercial ship can be known.

\section{RESULTS}

In this part, the authors would like to discuss about critical path with PDM, so that the expected time can be in accordance with the planning of the 60 meters commercial ship construction

The time and cost plan data of a 60 meter commercial ship construction project was for 314 days. The data on the schedule and duration of the 60 meter commercial ship building planning project along with the time duration are presented in table 1 and the cost budget design data are listed in table 2 . 
Table. 1 Schedule and Duration of the 60 Meter Commercial Ship Construction Project

\begin{tabular}{|c|c|c|c|c|}
\hline No & Activity & $\begin{array}{c}\text { Duration } \\
\text { (Day) }\end{array}$ & Start & Finish \\
\hline & Hull Construction & & $10 / 10 / 2016$ & $06 / 06 / 2017$ \\
\hline 1 & Hull Fabrication & 70 & $10 / 10 / 2016$ & $15 / 01 / 2017$ \\
\hline 2 & Hull Sub Assembly & 104 & $18 / 10 / 2016$ & $11 / 03 / 2017$ \\
\hline 3 & Hull Assembly & 137 & $26 / 10 / 2016$ & $06 / 05 / 2017$ \\
\hline \multirow[t]{2}{*}{4} & Hull Erection & 137 & $25 / 11 / 2016$ & $06 / 06 / 2017$ \\
\hline & Painting anode Catodic Protection & & $01 / 10 / 2016$ & $22 / 05 / 2017$ \\
\hline 5 & Blasting and Painting Raw Material & 48 & $10 / 10 / 2016$ & $15 / 12 / 2016$ \\
\hline 6 & Block Blasting and Painting & 137 & $15 / 11 / 2016$ & $31 / 05 / 2017$ \\
\hline 7 & Finishing Hull & 27 & $06 / 06 / 2017$ & $12 / 07 / 2017$ \\
\hline 8 & Painting Outfitting & 79 & $06 / 06 / 2017$ & $22 / 09 / 2017$ \\
\hline \multirow[t]{2}{*}{9} & Anode & 19 & $14 / 03 / 2017$ & $07 / 04 / 2017$ \\
\hline & Hull Outfitting & & $10 / 10 / 2016$ & $05 / 10 / 2017$ \\
\hline 10 & Seat and Foundation & 86 & $10 / 10 / 2016$ & $07 / 02 / 2017$ \\
\hline 11 & Deck Machinery and Equipment & 86 & $07 / 02 / 2017$ & $07 / 06 / 2017$ \\
\hline \multirow[t]{2}{*}{12} & Interior & 171 & $07 / 02 / 2017$ & $05 / 10 / 2017$ \\
\hline & Machinery Outfitting & & $10 / 10 / 2016$ & $05 / 10 / 2017$ \\
\hline 13 & Piping System Including Ducting & 181 & $10 / 10 / 2016$ & $20 / 06 / 2017$ \\
\hline 14 & Shafting & 51 & $20 / 06 / 2017$ & $30 / 08 / 2017$ \\
\hline \multirow[t]{2}{*}{15} & Machinery Outfitting & 25 & $31 / 08 / 2017$ & $05 / 10 / 2017$ \\
\hline & Electric, Electronic Outfitting & & $10 / 10 / 2016$ & $05 / 10 / 2017$ \\
\hline 16 & Cabling & 172 & $10 / 10 / 2016$ & $07 / 06 / 2017$ \\
\hline 17 & Power Supply & 172 & $02 / 11 / 2016$ & $01 / 07 / 2017$ \\
\hline 18 & Illuminiation & 172 & $26 / 11 / 2016$ & $26 / 07 / 2017$ \\
\hline 19 & $\begin{array}{l}\text { Communication and } \\
\text { Instrumentation }\end{array}$ & 178 & $21 / 12 / 2016$ & $18 / 08 / 2017$ \\
\hline 20 & Computing and Information & 172 & $13 / 01 / 2017$ & $12 / 09 / 2017$ \\
\hline \multirow[t]{2}{*}{21} & Nautical and Radio & 172 & $07 / 02 / 2017$ & $05 / 10 / 2017$ \\
\hline & Function and Commisioning & & $05 / 10 / 2017$ & $30 / 10 / 2017$ \\
\hline 22 & Machinery Commisioning & 9 & $05 / 10 / 2017$ & $18 / 10 / 2017$ \\
\hline 23 & Equipment Commisioning & 8 & $10 / 10 / 2017$ & $21 / 10 / 2017$ \\
\hline 24 & Lighting Commisioning & 4 & $18 / 10 / 2017$ & $24 / 10 / 2017$ \\
\hline \multirow[t]{2}{*}{25} & System Control Commisioning & 4 & $24 / 10 / 2017$ & $30 / 10 / 2017$ \\
\hline & HAT and SAT & & $02 / 11 / 2017$ & $18 / 12 / 2017$ \\
\hline 26 & Harbour Acceptance Test & 31 & $02 / 11 / 2017$ & $16 / 12 / 2017$ \\
\hline \multirow[t]{2}{*}{27} & Yard Trial & 14 & $27 / 11 / 2017$ & $18 / 12 / 2017$ \\
\hline & Delivery & & $20 / 12 / 2017$ & $20 / 12 / 2017$ \\
\hline 28 & Delivery to Customer & 0 & $20 / 12 / 2017$ & $20 / 12 / 2017$ \\
\hline
\end{tabular}

Table. 2 Budget Plan

\begin{tabular}{|c|c|c|c|c|}
\hline \multirow{2}{*}{ No. } & & \multirow{2}{*}{ Job Description } & \multirow{2}{*}{\multicolumn{2}{|c|}{$\begin{array}{c}\text { Cost } \\
\text { (Thousands Rupiah) }\end{array}$}} \\
\hline & & & & \\
\hline \multirow[t]{5}{*}{$\mathbf{A}$} & \multicolumn{2}{|c|}{ Hull Construction } & & \\
\hline & 1 & Hull Fabrication & $\mathrm{Rp}$ & 46.176 .942 \\
\hline & 2 & Hull Sub Assembly & $\mathrm{Rp}$ & 6.244 .996 \\
\hline & 3 & Hull Assembly & $\mathrm{Rp}$ & 8.286 .629 \\
\hline & 4 & Hull Erection & $\mathrm{Rp}$ & 8.286 .629 \\
\hline \multirow[t]{6}{*}{ B } & \multicolumn{2}{|c|}{ Painting anode Catodic Protection } & & \\
\hline & 5 & Blasting and Painting Raw Material & $\mathrm{Rp}$ & 3.242 .594 \\
\hline & 6 & Block Blasting and Painting & $\mathrm{Rp}$ & 2.522 .018 \\
\hline & 7 & Finishing Hull & $\mathrm{Rp}$ & 1.621 .297 \\
\hline & 8 & Painting Outfitting & $\mathrm{Rp}$ & 4.743 .795 \\
\hline & 9 & Anode & $\mathrm{Rp}$ & 1.140 .913 \\
\hline
\end{tabular}




\begin{tabular}{|c|c|c|c|c|}
\hline \multirow{2}{*}{ No. } & \multirow{2}{*}{\multicolumn{2}{|c|}{ Job Description }} & \multirow{2}{*}{\multicolumn{2}{|c|}{$\begin{array}{c}\text { Cost } \\
\text { (Thousands Rupiah) }\end{array}$}} \\
\hline & & & & \\
\hline \multirow[t]{4}{*}{$\mathbf{C}$} & \multicolumn{2}{|c|}{ Hull Outfitting } & & \\
\hline & 10 & Seat and Foundation & $\mathrm{Rp}$ & 5.224 .179 \\
\hline & 11 & Deck Machinery and Equipment & $\mathrm{Rp}$ & 5.224 .179 \\
\hline & 12 & Interior & $\mathrm{Rp}$ & 10.388 .311 \\
\hline \multirow[t]{4}{*}{ D } & \multicolumn{2}{|c|}{ Machinery Outfitting } & & \\
\hline & 13 & Piping System Including Ducting & $\mathrm{Rp}$ & 10.928 .743 \\
\hline & 14 & Shafting & $\mathrm{Rp}$ & 3.122 .498 \\
\hline & 15 & Machinery Outfitting & $\mathrm{Rp}$ & 1.561 .249 \\
\hline \multirow[t]{7}{*}{$\mathbf{E}$} & \multicolumn{2}{|c|}{ Electric, Electronic Outfitting } & & \\
\hline & 16 & Cabling & $\mathrm{Rp}$ & 4.203 .363 \\
\hline & 17 & Power Supply & $\mathrm{Rp}$ & 4.563 .651 \\
\hline & 18 & Illuminiation & $\mathrm{Rp}$ & 4.383 .507 \\
\hline & 19 & Communication and Instrumentation & $\mathrm{Rp}$ & 4.743 .795 \\
\hline & 20 & Computing and Information & $\mathrm{Rp}$ & 4.803 .843 \\
\hline & 21 & Nautical and Radio & $\mathrm{Rp}$ & 3.963 .171 \\
\hline \multirow[t]{5}{*}{$\mathbf{E}$} & \multicolumn{2}{|c|}{ Function and Commisioning } & & \\
\hline & $\overline{22}$ & Machinery Commisioning & $\mathrm{Rp}$ & 600.480 \\
\hline & 23 & Equipment Commisioning & $\mathrm{Rp}$ & 540.432 \\
\hline & 24 & Lighting Commisioning & $\mathrm{Rp}$ & 120.096 \\
\hline & 25 & System Control Commisioning & $\mathrm{Rp}$ & 420.336 \\
\hline \multirow[t]{3}{*}{$\mathbf{F}$} & \multicolumn{2}{|c|}{ HAT and SAT } & & \\
\hline & 26 & Harbour Acceptance Test & $\mathrm{Rp}$ & 1.921 .537 \\
\hline & 27 & Yard Trial & $\mathrm{Rp}$ & 900.721 \\
\hline \multirow[t]{3}{*}{ G } & \multicolumn{2}{|c|}{ Delivery } & & \\
\hline & 28 & Delivery to Customer & $\mathrm{Rp}$ & 120.096 \\
\hline & \multicolumn{2}{|c|}{ TOTAL } & $\mathrm{Rp}$ & 50.000 .000 \\
\hline
\end{tabular}

\section{Gantt Chart Planning Data for 60 Meter} Commercial Ship Construction Project

Making a planning schedule for a 60 meter commercial ship construction project used the Gantt Chart to determine when the activities / activities were started, postponed and completed.

The plan schedule of a 60 meter commercial ship construction project can be seen in the following figure below: 


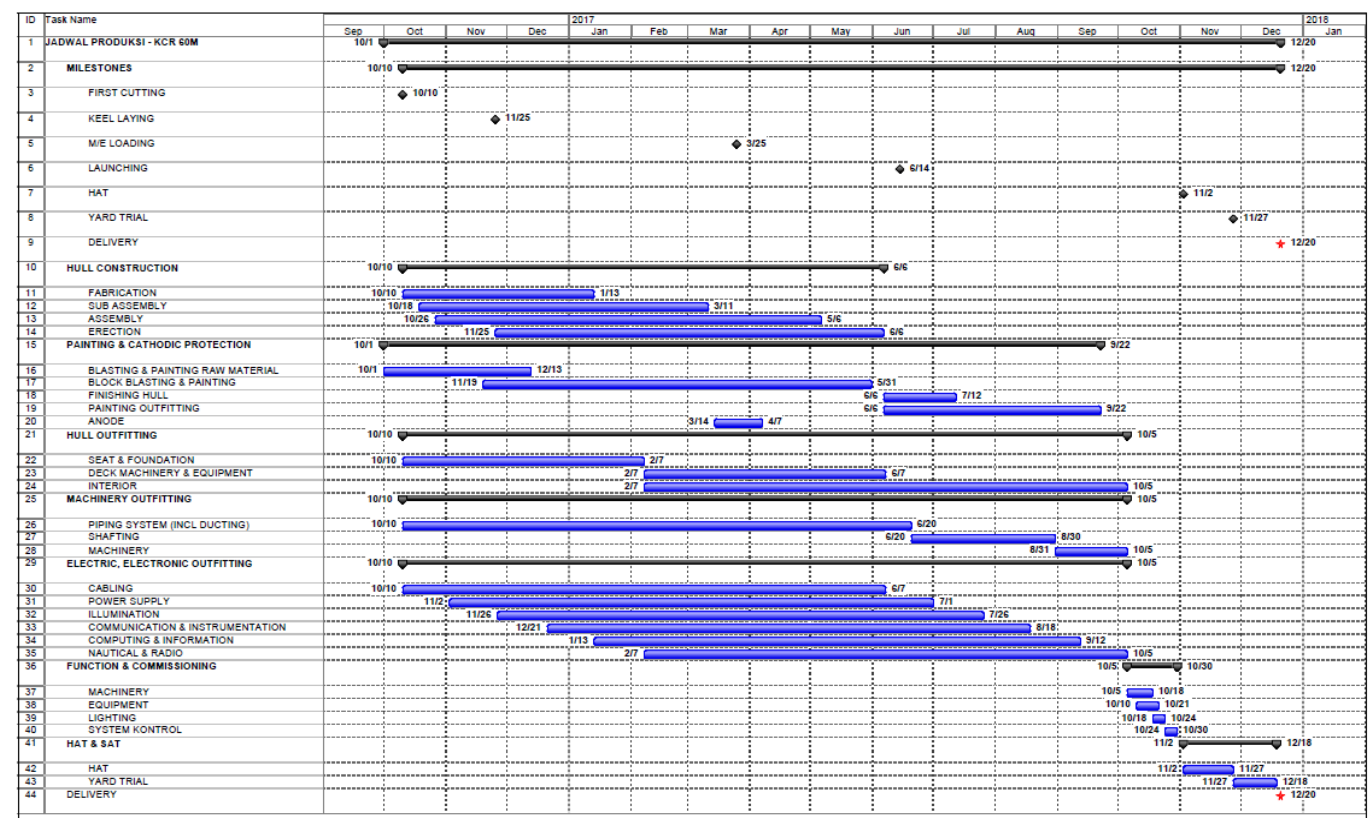

Fig. 5 Gantt Chart Planning Data for 60 Meter Commercial Ship Construction Project

\section{DISCUSSION}

In this study, the PDM method used one determinant number and four constraints. The four constraints in the Precedence Diagram Method (PDM) are important components in the formation of its network. In addition, the four constraints give different

Table. 3 Determination the Construction of the 60 Meter Commercial Ship Construction Project

\begin{tabular}{|c|l|l|}
\hline No & \multicolumn{1}{|c|}{ The Activities } & \multicolumn{1}{c|}{ Konstrain } \\
\hline 1 & Hull Fabrication & - \\
\hline 2 & Hull Sub Assembly & $\mathrm{SS}(1-2)=6$ \\
\hline 3 & Hull Assembly & $\mathrm{SS}(2-3)=6$ \\
\hline 4 & Hull Erection & $\mathrm{SS}(3-4)=22$ \\
\hline 5 & Blasting and Painting Raw Material & - \\
\hline 6 & Block Blasting and Painting & $\mathrm{SS}(5-6)=30$ \\
\hline 7 & Finishing Hull & $\mathrm{FS}(9-7)=42$ \\
\hline & & $\mathrm{FS}(3-7)=22$ \\
\hline & & $\mathrm{FS}(4-7)=0$ \\
\hline & & $\mathrm{FS}(6-7)=4$ \\
\hline 8 & Painting Outfitting & $\mathrm{FS}(4-8)=0$ \\
\hline & & $\mathrm{FS}(6-8)=4$ \\
\hline 9 & Anode & $\mathrm{FS}(2-9)=2$ \\
\hline 10 & Seat and Foundation & - \\
\hline 11 & Deck Machinery and Equipment & $\mathrm{FS}(10-11)=0$ \\
\hline 12 & Interior & $\mathrm{FS}(10-12)=0$ \\
\hline 13 & Piping System Including Ducting & - \\
\hline 14 & Shafting & $\mathrm{FS}(11-14)=9$ \\
\hline & & $\mathrm{FS}(13-14)=0$ \\
\hline 15 & Machinery Outfitting & $\mathrm{FS}(7-15)=37$ \\
\hline & & $\mathrm{SS}(8-15)=63$ \\
\hline & & $\mathrm{FS}(14-15)=1$ \\
\hline 16 & Cabling & \\
\hline 17 & Power Supply & $\mathrm{SS}(16-17)=17$ \\
\hline 18 & Illuminiation & $\mathrm{SS}(16-18)=35$ \\
\hline 19 & Communication and Instrumentation & $\mathrm{SS}(16-19)=52$ \\
\hline
\end{tabular}
display of precedent diagrams as nodes. determination on the 60 meter commercial ship construction project can be seen in the following table:

The results of the construction data 


\begin{tabular}{|l|l|l|}
\hline No & \multicolumn{1}{|c|}{ The Activities } & \multicolumn{1}{|c|}{ Konstrain } \\
\hline 20 & Computing and Information & $\mathrm{SS}(16-20)=70$ \\
\hline 21 & Nautical and Radio & $\mathrm{SS}(16-21)=86$ \\
\hline 22 & Machinery Commisioning & $\mathrm{FS}(12-22)=0$ \\
\hline & & $\mathrm{FS}(15-22)=0$ \\
\hline 23 & Equipment Commisioning & $\mathrm{FS}(16-23)=89$ \\
\hline & & $\mathrm{FS}(17-23)=72$ \\
\hline & & $\mathrm{FS}(18-23)=54$ \\
\hline & & $\mathrm{FS}(19-23)=31$ \\
\hline & & $\mathrm{FS}(20-23)=20$ \\
\hline & & $\mathrm{FS}(21-23)=4$ \\
\hline 24 & Lighting Commisioning & $\mathrm{SS}(23-24)=6$ \\
\hline 25 & System Control Commisioning & $\mathrm{FS}(24-25)=0$ \\
\hline 26 & Harbour Acceptance Test & $\mathrm{FS}(22-26)=11$ \\
\hline & & $\mathrm{FS}(23-26)=9$ \\
\hline & & $\mathrm{FS}(25-26)=3$ \\
\hline 27 & Yard Trial & $\mathrm{SS}(26-27)=18$ \\
\hline 28 & Delivery to Customer & $\mathrm{FS}(27-28)=2$ \\
\hline
\end{tabular}

Table. 4 Table of Activities for Construction of a 60 Meter Commercial Ship Construction Project

\begin{tabular}{|c|c|c|c|c|}
\hline No & Job Description & $\begin{array}{c}\text { Activity } \\
\text { Code }\end{array}$ & Initial Activity & $\begin{array}{c}\text { Duration } \\
\text { (Day) }\end{array}$ \\
\hline & Hull Construction & & & \\
\hline 1 & Hull Fabrication & 1 & - & 70 \\
\hline 2 & Hull Sub Assembly & 2 & 1 & 104 \\
\hline 3 & Hull Assembly & 3 & 2 & 137 \\
\hline \multirow[t]{2}{*}{4} & Hull Erection & 4 & 3 & 137 \\
\hline & Painting anode Catodic Protection & & & \\
\hline 5 & Blasting and Painting Raw Material & 5 & - & 48 \\
\hline 6 & Block Blasting and Painting & 6 & 5 & 137 \\
\hline 7 & Finishing Hull & 7 & $3,4,6,9$ & 27 \\
\hline 8 & Painting Outfitting & 8 & 4,6 & 79 \\
\hline \multirow[t]{2}{*}{9} & Anodizing & 9 & 2 & 19 \\
\hline & Hull Outfitting & & & \\
\hline 10 & Seat and Foundation & 10 & - & 86 \\
\hline 11 & Deck Machinery and Equipment & 11 & 10 & 86 \\
\hline \multirow[t]{2}{*}{12} & Interior & 12 & 10 & 171 \\
\hline & Machinery Outfitting & & & \\
\hline 13 & Piping System Including Ducting & 13 & - & 181 \\
\hline 14 & Shafting & 14 & 11,13 & 51 \\
\hline \multirow[t]{2}{*}{15} & Machinery Outfitting & 15 & 7.8 .14 & 25 \\
\hline & Electric, Electronic Outfitting & & & \\
\hline 16 & Cabling & 16 & - & 172 \\
\hline 17 & Power Supply & 17 & 16 & 172 \\
\hline 18 & Illuminiation & 18 & 16 & 172 \\
\hline 19 & Communication and Instrumentation & 19 & 16 & 178 \\
\hline 20 & Computing and Information & 20 & 16 & 172 \\
\hline \multirow[t]{2}{*}{21} & Nautical and Radio & 21 & 16 & 172 \\
\hline & Function and Commisioning & & & \\
\hline 22 & Machinery Commisioning & 22 & 12,15 & 9 \\
\hline 23 & Equipment Commisioning & 23 & $16,17,18,19,20,21$ & 8 \\
\hline 24 & Lighting Commisioning & 24 & 23 & 4 \\
\hline \multirow[t]{2}{*}{25} & System Control Commisioning & 25 & 24 & 4 \\
\hline & HAT and SAT & & & \\
\hline 26 & Harbour Acceptance Test & 26 & $22,23,25$ & 31 \\
\hline \multirow[t]{2}{*}{27} & Yard Trial & 27 & 26 & 14 \\
\hline & Delivery & & & \\
\hline 28 & Delivery to Customer & 28 & 27 & 0 \\
\hline
\end{tabular}


Based on the series of project activities in the table above with the addition of constrains provisions in table 4, they were then described in the form of a networking planning diagram using activity signs in the activity on node (AON) model as shown below:

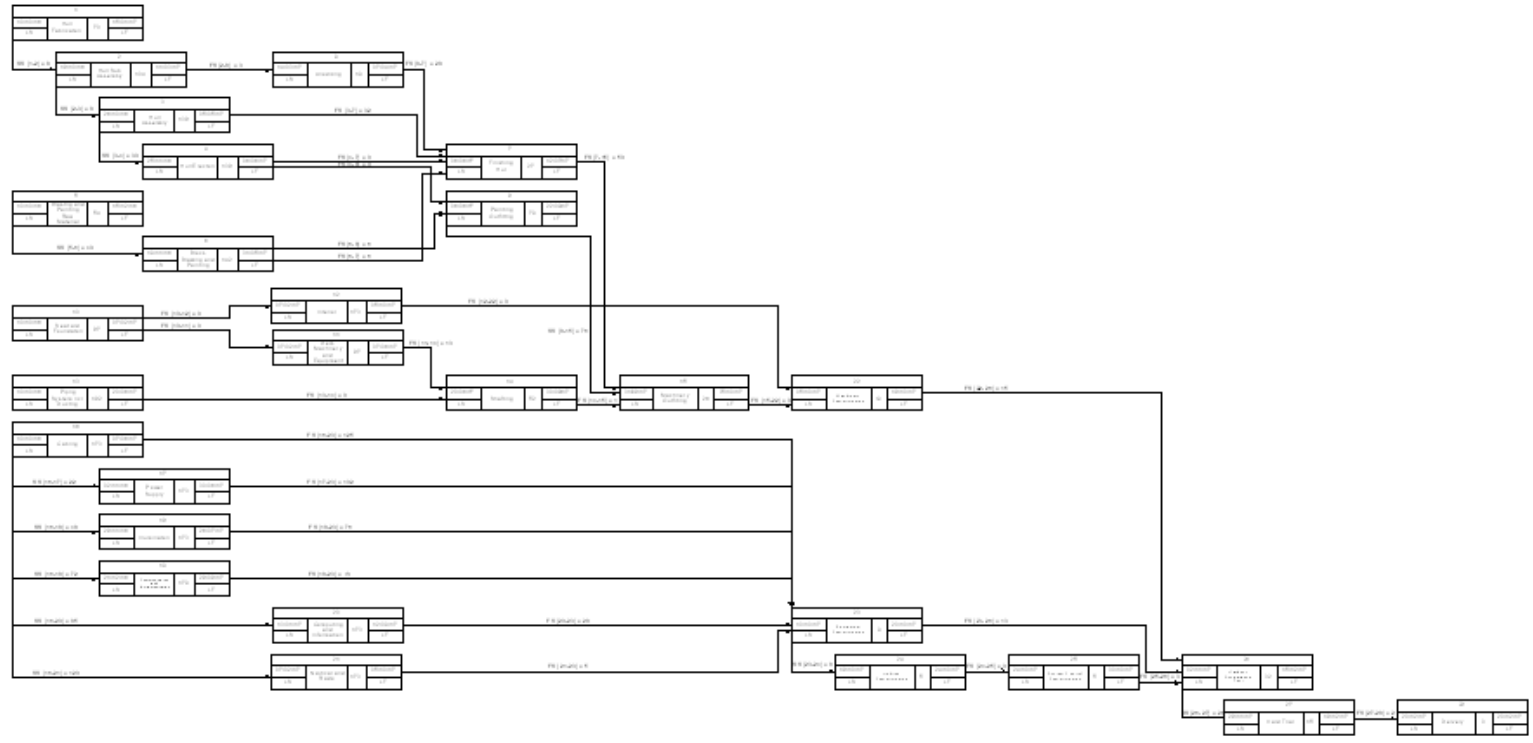

Fig. 6 Activity On Node of 60 Meter Commercial Ship Construction Project

After the Time Schedule was known, the next step was the researcher to perform the PDM calculation to find out the project completion time. The PDM it consists of two stages, namely forward pass and backward pass.

1. Forward Pass

○ Activity 1

$$
\begin{array}{ll}
\mathrm{ES}(1) & =0 \\
\mathrm{EF}(1) & =0+70=70
\end{array}
$$

○ Activity 2

ES (2) $\quad=0+6=6$

$\mathrm{EF}(2) \quad=6+104=110$

○ Activity 3

ES (3) $\quad=6+6=12$

$\mathrm{EF}(3) \quad=12+137=149$

○ Activity 4

ES (4) $\quad=12+22=34$

$\mathrm{EF}(4) \quad=34+137=171$

○ Activity 5

ES (5) $\quad=0$

$\mathrm{EF}(5) \quad=0+48=48$

○ Activity 6

$\mathrm{ES}=0+30=30$

$\mathrm{EF}=30+137=167$

○ Activity 7

ES (7) $\quad=130+42=172$

$=149+22=171$

$$
=171+0=171
$$$$
=167+4=171
$$

$\mathrm{EF}(7) \quad=172+27=199$

○ Activity 8

ES (8) $\quad=171+0=171$

$$
=167+4=170
$$

$\mathrm{EF}(8) \quad=171+79=250$

○ Activity 9

ES (9) $\quad=110+2=112$

$\mathrm{EF}(9) \quad=112+18=130$

- Activity 10

$\operatorname{ES~(10)~}=0$

$\mathrm{EF}(10)=0+86=86$

○ Activity 11

ES (11) $=87+0=86$

$\mathrm{EF}(11) \quad=86+86=172$

○ Activity 12

$\operatorname{ES}(12)=86+0=86$

$\mathrm{EF}(12)=86+171=257$

- Activity 13

$\operatorname{ES~(13)}=0$

$\mathrm{EF}(13) \quad=0+181=181$

○ Activity 14

ES (14) $\quad=172+9=181$

$=181+0=181$

$\mathrm{EF}(14) \quad=181+51=232$

○ Activity 15

ES (15) $=199+36=235$

$=171+62=233$ 


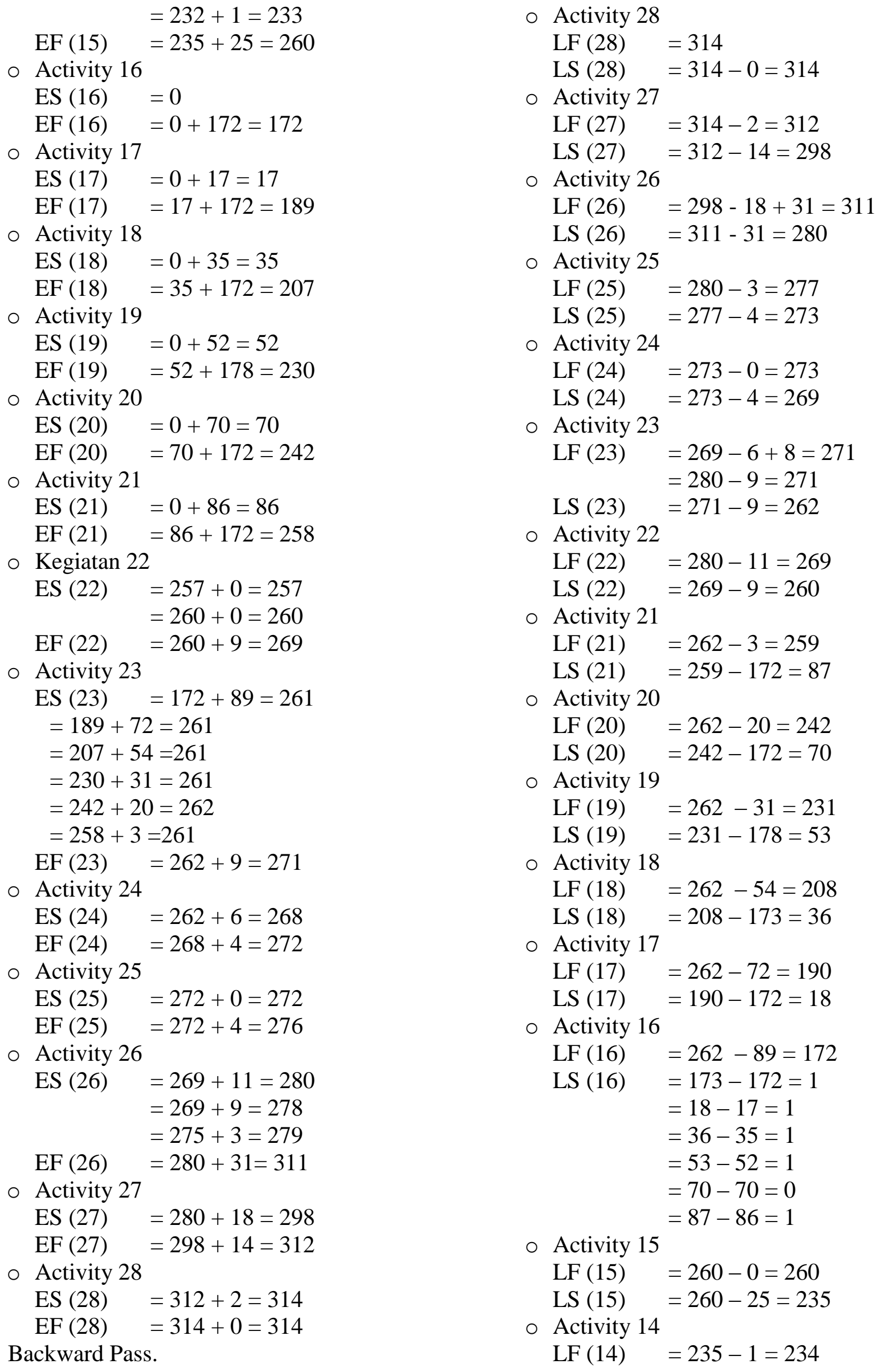


$\operatorname{LS}(14) \quad=234-51=183$

○ Activity 13

LF (13) $\quad=183-0=183$

LS (13) $\quad=183-182=1$

○ Activity 12

LF (12) $=260-0=260$

LS (12) $=260-171=89$

○ Activity 11

LF (11) $=183-9=174$

LS (11) $\quad=174-87=86$

○ Activity 10

LF (10) $\quad=87-0=87$

$$
=89-0=89
$$

LS (10) $\quad=87-86=1$

○ Activity 9
LF (9)
$=172-42=130$

LS (9)

$=130-18=112$

○ Activity 8

$\mathrm{LF}(8) \quad=235-62+79=252$

LS (8) $=252-79=173$

○ Activity 7

LF (7) $\quad=235-36=199$

LS (7) $\quad=199-27=172$

○ Activity 6

LF (6) = $172-4=168$

Table. 5 Recapitulation of ES, EF, SL, LS and LF Value Calculation of the 60 Meter Commercial Ship Construction Project

\begin{tabular}{|c|c|c|c|c|c|c|}
\hline Activity Code & $\begin{array}{c}\text { Duration } \\
\text { (Day) }\end{array}$ & ES & EF & SL & LS & LF \\
\hline $\mathbf{1}$ & $\mathbf{7 0}$ & $\mathbf{0}$ & $\mathbf{7 0}$ & $\mathbf{0}$ & $\mathbf{0}$ & $\mathbf{7 0}$ \\
\hline $\mathbf{2}$ & $\mathbf{1 0 4}$ & $\mathbf{6}$ & $\mathbf{1 2 0}$ & $\mathbf{0}$ & $\mathbf{6}$ & $\mathbf{1 2 0}$ \\
\hline $\mathbf{3}$ & $\mathbf{1 3 7}$ & $\mathbf{1 2}$ & $\mathbf{1 4 9}$ & $\mathbf{0}$ & $\mathbf{1 2}$ & $\mathbf{1 4 9}$ \\
\hline $\mathbf{4}$ & $\mathbf{1 3 7}$ & $\mathbf{3 4}$ & $\mathbf{1 7 3}$ & $\mathbf{0}$ & $\mathbf{3 4}$ & $\mathbf{1 7 3}$ \\
\hline $\mathbf{5}$ & $\mathbf{4 8}$ & $\mathbf{0}$ & $\mathbf{4 8}$ & $\mathbf{0}$ & $\mathbf{0}$ & $\mathbf{4 8}$ \\
\hline 6 & 137 & 30 & 167 & 1 & 31 & 168 \\
\hline $\mathbf{7}$ & $\mathbf{2 7}$ & $\mathbf{1 7 2}$ & $\mathbf{1 9 9}$ & $\mathbf{0}$ & $\mathbf{1 7 2}$ & $\mathbf{1 9 9}$ \\
\hline 8 & 79 & 171 & 250 & 2 & 173 & 252 \\
\hline $\mathbf{9}$ & $\mathbf{1 9}$ & $\mathbf{1 1 2}$ & $\mathbf{1 3 0}$ & $\mathbf{0}$ & $\mathbf{1 1 2}$ & $\mathbf{1 3 0}$ \\
\hline 10 & 86 & 0 & 86 & 1 & 1 & 87 \\
\hline 11 & 86 & 86 & 172 & 1 & 87 & 173 \\
\hline 12 & 171 & 86 & 257 & 1 & 87 & 258 \\
\hline 13 & 181 & 0 & 181 & 1 & 1 & 183 \\
\hline 14 & 51 & 181 & 232 & 2 & 183 & 234 \\
\hline $\mathbf{1 5}$ & $\mathbf{2 5}$ & $\mathbf{2 3 5}$ & $\mathbf{2 6 0}$ & $\mathbf{0}$ & $\mathbf{2 3 5}$ & $\mathbf{2 6 0}$ \\
\hline $\mathbf{1 6}$ & $\mathbf{1 7 2}$ & $\mathbf{0}$ & $\mathbf{1 7 2}$ & $\mathbf{0}$ & $\mathbf{0}$ & $\mathbf{1 7 2}$ \\
\hline 17 & 172 & 17 & 189 & 1 & 18 & 190 \\
\hline 18 & 172 & 35 & 207 & 1 & 36 & 208 \\
\hline 19 & 178 & 52 & 230 & 1 & 53 & 231 \\
\hline $\mathbf{2 0}$ & $\mathbf{1 7 2}$ & $\mathbf{7 0}$ & $\mathbf{2 4 2}$ & $\mathbf{0}$ & $\mathbf{7 0}$ & $\mathbf{2 4 2}$ \\
\hline 21 & 172 & 86 & 258 & 1 & 87 & 259 \\
\hline $\mathbf{2 2}$ & $\mathbf{9}$ & $\mathbf{2 6 0}$ & $\mathbf{2 6 9}$ & $\mathbf{0}$ & $\mathbf{2 6 0}$ & $\mathbf{2 6 9}$ \\
\hline $\mathbf{2 3}$ & $\mathbf{8}$ & $\mathbf{2 6 2}$ & $\mathbf{2 7 1}$ & $\mathbf{0}$ & $\mathbf{2 6 2}$ & $\mathbf{2 7 1}$ \\
\hline 24 & 4 & 268 & 272 & 1 & 269 & 273 \\
\hline 25 & 4 & 272 & 276 & 1 & 273 & 277 \\
\hline & & & & & & \\
\hline
\end{tabular}




\begin{tabular}{|c|c|c|c|c|c|c|}
\hline Activity Code & $\begin{array}{c}\text { Duration } \\
\text { (Day) }\end{array}$ & ES & EF & SL & LS & LF \\
\hline 26 & 31 & 280 & 311 & 0 & 280 & 311 \\
\hline 27 & 14 & 298 & 312 & 0 & 298 & 312 \\
\hline 28 & 0 & 314 & 314 & 0 & 314 & 314 \\
\hline
\end{tabular}

Based on Table 5, the critical path from the PDM calculation was obtained. The critical path is the path through which critical work passes, namely the work that has the earliest start time equals with the last start

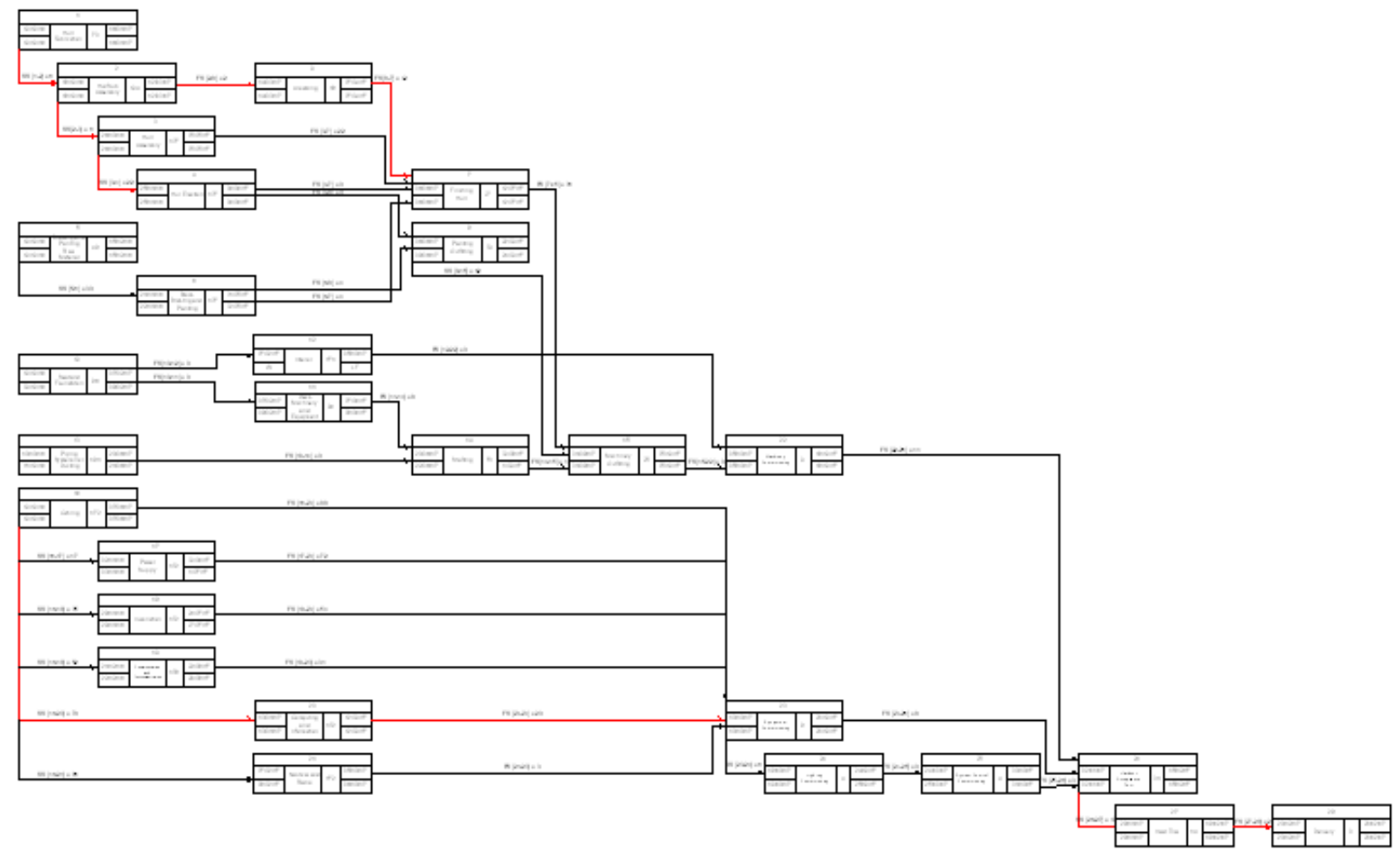

Fig. 7 PDM critical track on the 60 Meter Commercial Ship Construction Project

\section{CONCLUSION}

Based on the calculations obtained, activity Hull Fabrication, Hull Sub Assembly, Hull Assembly, Hull Erection, Blasting and Painting Raw Material, Finishing Hull, Anode, Machinery Outfitting, Cabling, Computing and Information, Machinery Commisioning, Equipment Commisioning, Harbour Acceptance Test, Yard Trial, Delivery to Customer are critical paths. Thus, control and supervision is more stringent in carrying out the activities of the 60 meter commercial ship building project activitiesthan the contractor on each activity to avoid delays so that the project can be completed on time. time $(\mathrm{ES}=\mathrm{LS})$ and the earliest completion time equals with the latest finish time $(\mathrm{EF}=$ LF). The critical path on PDM can be seen in the following figure: 
Alecu, F. (2014). Cost Control and Performance Review of Software Projects by Using the Earned Value Management. Oeconomics of Knowledge , 2-6.

Andawei, M.-E. M. (2014). Extension of Time Determination in Construction Projects in Nigeria: The Critical Path Method. The International Journal of Engineering And Science (IJES) , 48-51.

Arica Dwi Susanto, A. A. (2018). The Optimization of Multipurpose Building Development on Project Scheduling Using Precedence Diagram Method (PDM). ASRO JOURNAL-STTAL, 9 (1), 1-7.

Balla, G. T. (1995). Critical path analysis for the management of fractured neck of femur. AUSTRALIAN JOURNAL OF PUBLIC HEALTH , 155-159.

Czarnigowska, A. (2008). Earned value method as a tool for project control. Budownictwo i Architektura 3 , 15-32.

Daniel Castro-Lacouture, A. G.-J. (2009). Contruction Project Scheduling with Time, Cost and Material Restrictions Using Fuzzy Mathematical Models and Critical Path Method . JOURNAL OF CONSTRUCTION ENGINEERING AND MANAGEMENT , 1096-1104.

Dwaikat, L. N. (2016). Measuring the Actual Energy Cost Performance of Green Buildings: A Test of the Earned Value Management Approach. Energies journal , 1-20.

Fatemeh Nouban, N. G. (2017). The Factors Affecting The Methods of Construction Projects Scheduling: An State of The Art and Overview. Asian Journal of Natural \& Applied Sciences, 6 (4), 114-122.

Ikhtisholiyah. (2017). analysis of the application of time and cost management on the construction project of electric engineering building of polytechnic industry of madura (poltera). Zeta-Math Journal , 14-21.
K.K.Khandelwal, D. B. (2002). PROJECT PLANNING AND CONTROL WITH PERT AND CPM. New Delhi: LAXMI PUBLICATIONS (P) LTD.

Kim, S.-G. (2012). CPM Schedule Summarizing Function of the Beeline Diagram Method. Journal of Asian Architecture and Building Engineering, 367-374.

M, S. (2015). Traditional Critical Path Method versus Critical Chain Project Management: A Comparative View. International Journal of Economics \& Management Sciences, 1-6.

M. Hayuningtyas, T. D. (2018). System analysis for technology transfer readiness assessment of horticultural postharvest. International Conference on Industrial and System Engineering (IConISE) (hal. 1-7). Bogor: IOP Publishing.

Mazlum, M. (2015). CPM, PERT and Project Management With Fuzzy Logic Technique and Implementation On A Business. 4th International Conference on Leadership, Technology, Innovation and Business Management (hal. 348-357). Istanbul: Elsevier Ltd.

Muhammad Kholil, B. N. (2018). Scheduling of House Development Projects with CPM and PERT Method for Time Efficiency (Case Study: House Type 36). IOP Conf. Series: Earth and Environmental Science (hal. 1-8). jakarta: IOP Publishing.

Nafkha, R. (2016). THE CRITICAL PATH METHOD IN ESTIMATING PROJECT DURATION. Information Systems in Management, 78-87.

R.Lin, H. J. (2009). A fuzzy pert approach to evaluate plant construction project scheduling risk under uncertain resources capacity. Journal of Industrial Engineering and Management, 31-47. 
Shailla. (2014). Comparative Study of Management Operation System Techniques (MOST) and CPM in Construction Scheduling. International Journal of Engineering Trends and Technology (IJETT), 371-379.

Shan, Y. (2014). Integration of Building Information Modeling and Critical Path Method Schedules to Simulate the Impact of Temperature and Humidity at the Project Level. buildings jounal, 295-319.

Silvianita, R. F. (2018). Fast Missile Boat Project Planning using CPM and What If Analysis Method. IOP Conf. Series: Earth and Environmental Science (hal. 1-6). jakarta: IOP Publishing.

Wilson, B. (2013). Earned Value Management Systems: Challenges and Future Direction. Journal of Integrated Enterprise Systems , 917.

Wong, Y. (1964). Critical Path Analysis for New Product Planning. Journal of Marketing , 53-59.

Y. Arslan, H. B. (2017). Planning and Monitoring of industrial punch development processes . Journal of Engineering Research and Applied Science , 615-622.

\section{Creative Commons Attribution License 4.0 (Attribution 4.0 International, CC BY 4.0)}

This article is published under the terms of the Creative Commons Attribution License 4.0

https://creativecommons.org/licenses/by/4.0/deed.en_US 